\title{
Measurement of oocyte temporal maturation process by means of a simple optical micro-system
}

\author{
Florie Vidberg a,1, Rabah Zeggari ${ }^{\mathrm{b}, 2}$, Christian Pieralli $^{\mathrm{b}, 2}$, Clotilde Amiot ${ }^{\mathrm{a}, 1}$, \\ Christophe Roux ${ }^{\mathrm{a}, 1}$, Bruno Wacogne ${ }^{\mathrm{b}, *}$ \\ a Service de Génétique Histologie Biologie du Développement et de la Reproduction, É Clinical Investigation Center (CIC) IFR 133: IBCT, CHU St. Jacques, \\ IFR 133: IBCT Université de Franche-Comté, 25030 Besançon, France \\ b Institut FEMTO-ST, UMR CNRS 6174, Département d'Optique P.M. Duffieux, Faculté des Sciences et des Techniques, Route de Gray, 25030 Besançon Cedex, France
}

\begin{abstract}
We present a simple optical micro-system used to measure the transmission spectra of oocytes in order to qualify their maturation stage. Two applications of the device are possible: (i) the evaluation of the maturation stages of oocytes, and (ii) the development of a fertilization indicator. For the first applica-tion, GV, MI and MII oocytes were also analysed. Transmission spectra allow the 3 maturation stages to be identified but cannot be used to estimate the maturity of an unknown oocyte. Oocytes are subject to continuous development. This is why spectral separation of the 3 maturation stages cannot be made although they can be visually identified. However, the visual observation remains biologist-dependent. We therefore investigated the temporal maturation evolution of the oocytes in terms of transmission spectra and probability analysis. Results show that oocytes to be fertilized should not only be chosen in the MII stage, but also at the right time during the MII stage. This particular aspect requires further inves-tigation. However, spectral measurements could be used as a technique for monitoring the maturation evolution of the oocytes. Fertilized oocytes exhibiting fertilization abnormalities were also tested. The device proved to be an efficient fertilization indicator.
\end{abstract}

\section{Introduction}

The ability of an oocyte to be fertilized and to initiate embryo development strongly depends on its maturation stage. Oocyte maturity is defined by both nuclear and cytoplasmic maturities. The nuclear maturity can be estimated by observing oocytes with a microscope. This technique is commonly used in in vitro fertilization (IVF) centers in order to choose the oocytes to be fertilized by Intra-Cytoplasmic Sperm Injection (ICSI). However, the cytoplasmic maturity is more difficult to estimate and is somehow biologist-dependent. Only certain abnormalities can be detected by optical microscopy. Several attempts have been made to correlate various morphological parameters with successful ICSI. Conclusions concerning the importance of some morpholog-

\footnotetext{
* Corresponding author at: FEMTO-ST, Dprt of Optics P.M. Duffieux, Route de Gray, 25000 Besancon, France. Tel.: +33 3816663 88; fax: +33 381666423 .

E-mail addresses: florie.vidberg@hotmail.fr (F. Vidberg),

rabah.zeggari@femto-st.fr (R. Zeggari), christian.pieralli@univ-fcomte.fr (C. Pieralli), clotilde.amiot@univ-fcomte.fr (C. Amiot),

christophe.roux@univ-fcomte.fr (C. Roux), bruno.wacogne@univ-fcomte.fr (B. Wacogne).

1 Tel.: +33381218698; fax: +33381218668.

2 Tel.: +33 3816663 88; fax: +33 381666423 .
}

ical characteristics differ from one study to another (cytoplasm granularity, polar body appearance, zona pellucida appearance, perivitellin space dimension, etc.).

In the study presented in Ref. [1], oocyte quality was estimated via nuclear and cytoplasmic characteristics. Nuclear maturity has been defined by the extrusion of the first polar body and the cytoplasmic maturity by the homogeneity and the thin granularity of the cytoplasm. A correlation between oocyte quality and successful ICSI was shown. Equivalent correlations have been shown in other communications [2-4]. They concern the granularity of the cytoplasm, the presence of intra-cytoplasmic inclusions, the appearance of the first polar body and the dimension of the perivitellin space. However, such correlations have not been found in other studies [5-7]. Up to now, it seems very difficult to estimate oocyte quality in terms of morphological characteristics. This is probably due to the fact that these techniques rely on a visual observation of the oocytes which is highly biologist-dependent and therefore relatively subjective.

Since the dawn of IVF, certain technological developments have been studied. They mainly concern the preparation, the conditioning and the conservation of gametes, as well as the culture of oocytes and embryos [8]. Oocyte maturity has been investigated by means of mechanical measurements. These studies were mainly conducted on animal eggs [9-13]. To our knowledge, only one 


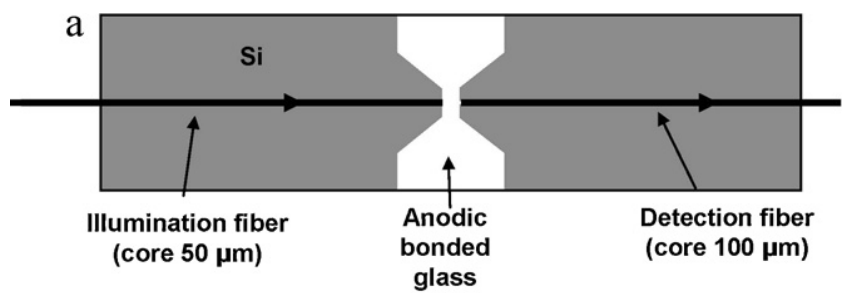

b
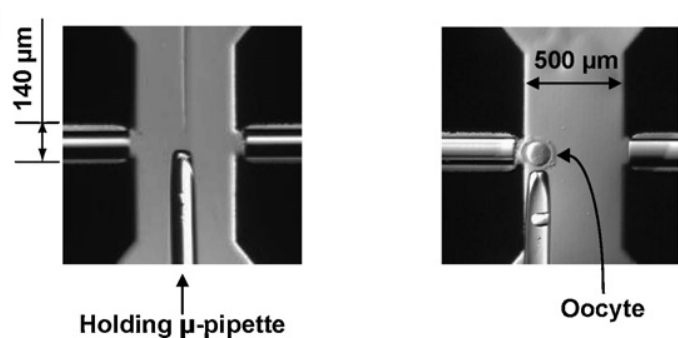

Fig. 1. Schematic diagram (a) and microscope views (b) of the device.

conference and one paper report experiments on human oocytes $[14,15]$.

Optical methods offer potential possibilities [16,17]. In this paper, we present a simple micro-system used to measure the absorption spectra of oocytes. It is based on work we presented recently where we showed that the transmission spectra of oocytes change according to their maturation stage [16]. This recent study was mainly devoted to the production of a micro-fluidic platform used to manipulate the oocytes. Specific structures had been designed to include optical sensors on the platform surface. However, control of the fluidic system was neither integrated nor automated. The system was therefore somewhat difficult to use and only a small number of oocytes were tested. This is why we produced a more convenient micro-system that can easily be used in IVF centers. With this system, and using a very low power white light source, it is possible to record the spectrum of the light propagated through the oocytes. Therefore, the transmission spectrum of the oocytes under test may be recorded.

\section{Materials and methods}

\subsection{Experimental set-up}

A schematic diagram as well as microscope views of the device are shown in Fig. 1. It consists of a micro-machined piece of silicon anodic bonded on to a glass substrate. The silicon part of the device is etched by means of Deep Reactive Ion Etching in an Alcatel machine with the standard Bosch process. The substrate material is Pyrex glass 7740. This glass is used because it exhibits a similar thermal extension coefficient to silicon. Only the silicon part of the device is micro-machined. The main features are two U-grooves into which optical fibers are stuck. In this way, optical fibers are perfectly aligned. Fibers are stuck with a bio-compatible glue by means of extra structures which are not shown in the figure. A specific pigtailing arrangement was designed in order to adjust the distance between the two fibers precisely $(500 \mu \mathrm{m})$. Indeed, dedicated micro-stops were etched as described in reference [16]. Fibers were conventional $125 \mu \mathrm{m}$ diameter fibers. The core of one fiber is $50 \mu \mathrm{m}$ in diameter, called the illumination fiber. The core of the second fiber (the collecting one) is $100 \mu \mathrm{m}$ in diameter. In this arrangement, oocytes are in contact with glass only. The silicon part of the system has been thermally oxidized in order to produce a thin glass layer at the surface of the silicon. Therefore, oocytes are in contact with the same material as micro-pipettes commonly used in ICSI. After the device was produced, sterilization was carried out by UV illumination. During this study, the decontamination of the experimental device was performed with an ionic surfactant solution and multiple washings with sterilized pure water. It is worth noting that if the device is considered to be used on a routine basis, any conventional sterilization method can be employed. Indeed, the device is made of glass, oxidized silicon and bio-compatible glue.

The space between the two fibers is used to position the oocytes. The position of the latter is manually controlled by means of the micro-manipulator arrangement used in IVF centers. The glass substrate allows the position of the oocytes to be controlled with a microscope. Fig. 1(b) shows microscope views of the device where an oocyte is placed in front of the illumination fiber. A low power white light source is used to illuminate the oocytes (Ocean Optics LS-1). The coupling efficiency between the white light source and the illumination fiber is very low. Therefore, the optical power incident on the oocytes does not exceed a few $\mathrm{mW}$. This is several orders of magnitude lower than the optical power of the microscope light. Consequently, our system does not overstress the oocytes. The light propagated through the oocytes is then launched into the collection fiber and directed to a microspectrometer (Ocean Optics USB 2000+). In this way, transmission spectra of the oocytes are easily recorded and the acquisition time of the data can be reduced to $3 \mathrm{~ms}$.

The transmission spectrum of the cell is recorded in two steps. The first step consists in recording the spectral response $I_{1}(\lambda)$ of the system with the culture medium only. We therefore have:

$I_{1}(\lambda)=I_{0}(\lambda) C_{1} R(\lambda)$

where $I_{0}(\lambda)$ is the intensity spectrum of the white light source; $C_{1}$ the coupling coefficient between the two fibers and $R(\lambda)$ is the spectral response of the spectrometer.

Here, we consider that the refractive index of the culture medium is constant from one measurement to another, i.e. $C_{1}$ is the constant.

The second step consists in recording the spectral response of the system when the oocyte is correctly trapped in the center of the two fibers. We have:

$I_{2}(\lambda)=I_{0}(\lambda) T(\lambda) C_{2} R(\lambda)$

where $T(\lambda)$ is the transmission spectrum of the oocyte and $C_{2}$ is the coupling coefficient between the two fibers, taking the convergence properties of the oocyte into account.

To begin with, we suppose that all the oocytes have the same diameter and refractive index. $C_{2}$ is therefore constant. The result of Eq. (2) divided by Eq. (1) is then proportional to the transmission spectrum of the oocyte:

$\frac{I_{2}(\lambda)}{I_{1}(\lambda)}=\frac{C_{2}}{C_{1}} T(\lambda)$

Transmission spectra are recorded in a wavelength window ranging from $380 \mathrm{~nm}$ to $940 \mathrm{~nm}$.

\subsection{Biological material}

Oocytes used in this study were excluded from Assisted Reproductive Technology (ART) programs. Their use has been approved by the clinical ethics committee of Besançon University Hospital on May 5th 2004. Analyses were conducted using two groups.

The first group consisted of non-injected oocytes. On the one hand, we used oocytes that are not meant to be injected because of their maturation stage $(\mathrm{GV}$ : germinal vesicle $=$ prophase of the first meiotic division, and MI: metaphase $1=$ germinal vesicle breakdown "GVBD"). On the other hand, we tested MII oocytes (metaphase 2 = with the presence of the first polar body). These are oocytes collected at a lower degree of maturity than those evolved 


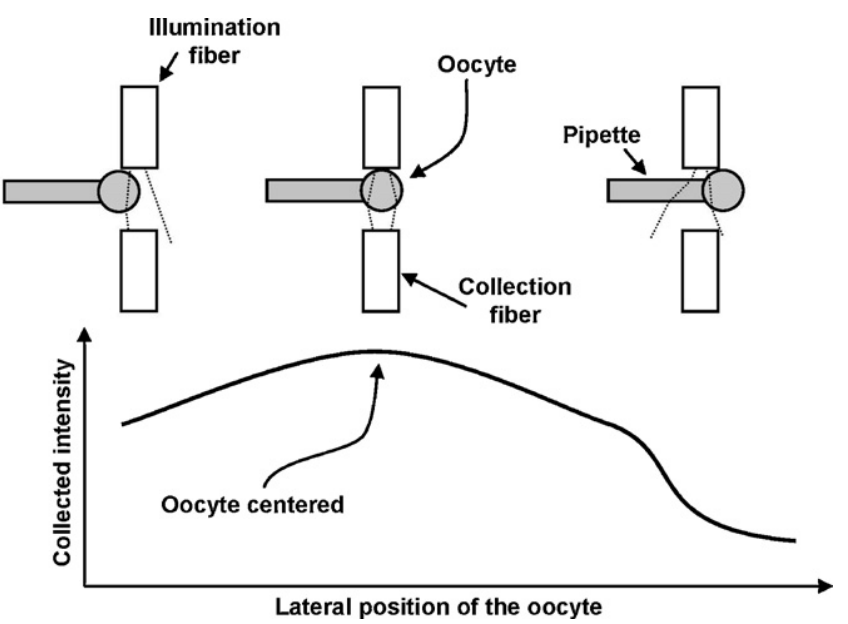

Fig. 2. Illustration of the lateral positioning of the oocyte on the fiber axis. The oocyte is correctly centered when the collected intensity is at the maximum threshold.

to the MII stage, or MII collected oocytes that have not been considered because of the lack of spermatozoon, and also oocytes issued from follicular reduction before intra-uterine insemination to prevent hyperstimulation and multiple pregnancies.

The second group consists of injected oocytes exhibiting fertilization abnormalities after ICSI, with presence of 3 pronuclei (3PN).

\subsection{Experimental protocol}

Oocytes from group 1 were collected during attempts at assisted reproductive technology (ART) by means of ICSI, or from follicular reduction. The complex cumulus-oocyte were individualized under a binocular microscope and transferred into a culture medium (Ferticult IVF medium $^{\mathrm{TM}}$, Fertipro, Belgium). After $90-120 \mathrm{~min}$ of in vitro culture at $37^{\circ} \mathrm{C}$, the cumulus was removed. This led to a perfect visualization of the oocytes under a microscope and allows their nuclear/meiotic maturation stage to be determined. Cumulus removal was performed in two steps:

- Enzymatic removal with a hyaluronidase solution (80 IU Hyaluronidase in Flushing, Fertipro, Belgium) for about 20 s.

- Mechanical removal by means of denudation micropipettes (SWEMED by Vitrolife, Sweden).

The oocytes were individually transferred into a 4 wells petri dish (Nunclon Delta SI, Nunc, Denmark) with $100 \mu$ l of culture medium (IVF30 ${ }^{\mathrm{TM}}$, Vitrolife, Sweden). Their maturation stage was defined by visual inspection with an inverted microscope.

Oocytes from group 2 were detected $17-24 \mathrm{~h}$ after fertilization by ICSI. They consisted of fertilized oocytes exhibiting fertilization abnormalities (3PN).

For both groups, $200 \mu$ l of IVF medium was deposited between the two fibers of the device. A first spectrum was recorded with the culture medium only $\left(I_{1}(\lambda)\right.$ in Eq. (1)). One oocyte was collected from the plastic box in $20 \mu$ l of IVF medium and deposited somewhere between the two fibers. The holding micropipette was then employed in order to place the oocyte precisely on the axis of the two fibers and in contact with the illumination fiber. The oocyte diameter $(150 \mu \mathrm{m})$ was comparable to the fiber diameter. Therefore, we consider that the right vertical position of the oocytes is obtained when both the fiber and the oocytes are in focus (Fig. 1(b)). The lateral positioning of the oocytes (on the fiber axis) is illustrated in Fig. 2 and can be summarized as follows. When the oocyte is slightly on the axis, a certain optical intensity is coupled into the collection fiber (on the left of the figure). When moving the oocyte
Table 1

Average characteristics of the transmission spectra for GV, MI and MII oocytes. The last three rows correspond to the Student's $t$-test.

\begin{tabular}{lll}
\hline & Minimum transmission (a.u.) & Minimum wavelength (nm) \\
\hline $\mathrm{GV}(n=57)$ & 0.34 & 464 \\
$\mathrm{MI}(n=70)$ & 0.43 & 451 \\
MII $(n=40)$ & 0.49 & 439 \\
$\mathrm{GV} /$ MI & $p<0.001$ & $p=0.01$ \\
$\mathrm{GV} /$ MII & $p<0.001$ & $p<0.01$ \\
MI/MII & $p=0.015$ & $p=0.045$ \\
\hline
\end{tabular}

toward the optical axis, the intensity increases up to a maximum (center of the figure). This maximum is reached when the oocyte is perfectly positioned on the optical axis and is due to the focusing properties of the oocyte that acts as a micro-lens. When the oocyte is further translated, the intensity starts to decrease until the point at which the holding micropipette masks the emitted light (on the right of the figure). When the oocyte is correctly positioned, a second spectrum is recorded $\left(I_{2}(\lambda)\right.$ (in Eq. (2)). The transmission spectrum of the oocytes is then computed according to Eq. (3).

Once the transmission spectrum is recorded, the oocytes are replaced in the IVF medium in the 4 wells petri dish. They are then incubated in a Sanyo $\mathrm{CO}_{2}$ incubator $\left(37^{\circ} \mathrm{C}, 5 \% \mathrm{CO}_{2}\right)$. After a while, any evolution (in terms of maturation) is controlled 4 and $17 \mathrm{~h}$ after the first maturity evaluation with the microscope. If the maturation stage of the oocyte evolves, another transmission spectrum is recorded.

Statistical comparisons were performed using the Student's $t$ test and $p<0.05$ was considered statistically significant. In order to better understand the nature of the evolution processes in the 3 classes, we investigated in more detail the probabilistic behavior of the optical data we recorded.

\section{Results}

\subsection{Experimental results for group 1}

This group consists of non-fertilized oocytes belonging to three maturation stages: GV, MI and MII. Overall, $57 \mathrm{GV}, 80 \mathrm{MI}$ and 40 MII oocytes were analysed. Not all the oocytes had been collected at the above mentioned classes. In fact, the $57 \mathrm{GV}$ oocytes had been collected at this degree of maturity. Among the 70 MI oocytes, 37 oocytes had been collected at this degree of maturity and 43 were issued from GV oocytes for which the maturity process continued during incubation. Among the $40 \mathrm{MII}$ oocytes, 15 had been collected at this degree of maturity, 14 were issued from MI oocytes and 11 were issued from GV oocytes. In other words, $75 \%$ of the oocytes collected as GV evolved to MI while $19 \%$ of them evolved up to MII. Also, 36\% of the oocytes collected as MI evolved to MII.

First of all, we calculated the average spectra corresponding to the 3 maturation stages. It can be seen in Table 1 that both values of the minimum transmissions and the corresponding wavelengths vary according to the maturity. The minimum transmissions increase from 0.34 (a.u.) for GV, to 0.43 (a.u.) for MI and to 0.49 (a.u.) for MII oocytes. At the same time, the wavelengths corresponding to these minima decrease from $464 \mathrm{~nm}$ for $\mathrm{GV}$, to 451 for MI and to 439 for MII oocytes.

Fig. 3(a) and (b) shows the Box and Whiskers diagrams for the 3 oocytes populations. Fig. 3(a) refers to the minimum transmission while Fig. 3(b) concerns the minimum wavelength. These diagrams represent several distribution or dispersion characteristics of the statistical series. They allow a particular characteristic (minimum transmission or wavelength) of populations of different sizes to be compared. It is clearly observed from this figure that the 3 maturation stages are well identified. At the same time, it is also obvious that these 3 stages overlap. This means that, although the classes 

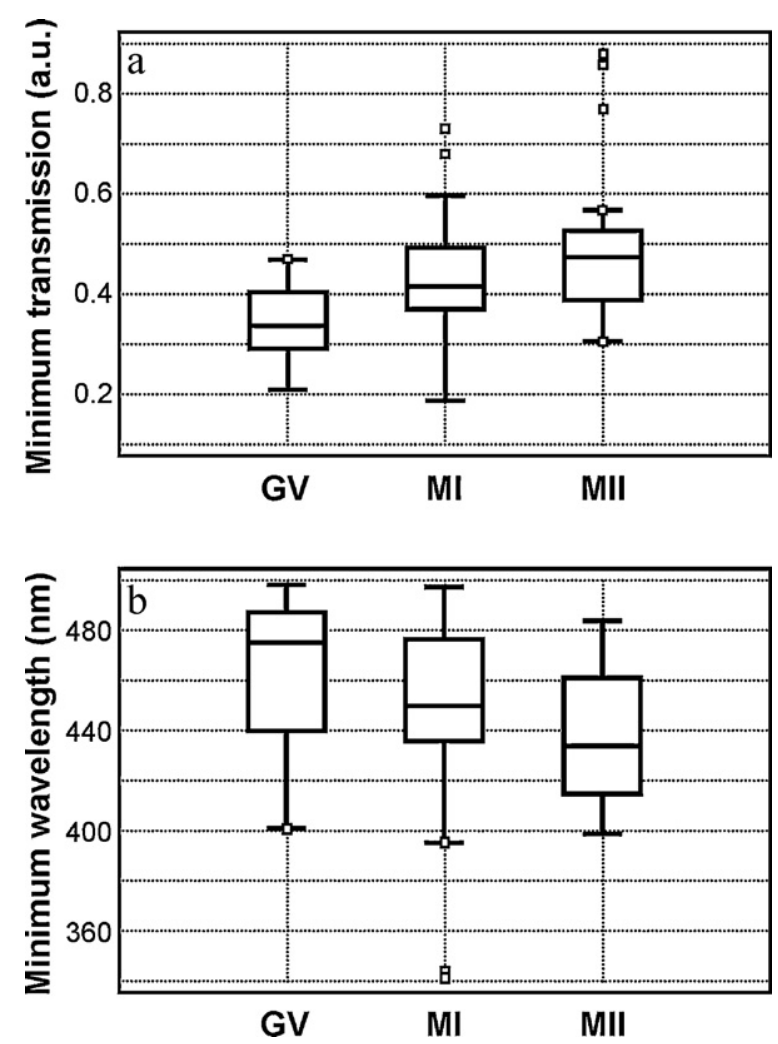

Fig. 3. Box and Whiskers diagrams for the three oocyte stages (GV, MI and MII). (a) refers to the minimum transmissions while (b) refers to the minimum wavelengths.

are identified, the analysis of the transmission spectra cannot be used in a blind test. We also compared the transmission spectra of GV oocytes that had evolved to the MI class with the spectra of GV oocytes without evolution. No significant differences between the spectra were observed. This was the same between MI and MII and between GV and MII.

\subsection{Experimental results with group 2}

Another possibility for exploiting the transmission spectra could be to study the actual shape of the spectra (by means of Fourier descriptors for example). This idea comes from the experimental results we obtained with fertilized oocytes corresponding to the second group of our study.

This group consists of fertilized oocytes for which abnormalities (3PN) were observed. Two cases arose. After fertilization, a 3PN can either lead to a segmentation process or not. The observation of segmentation means that fertilization occurred, the process was qualified as "successful" and embryo development starts. In this case, of course, development does not come to an end. On the contrary, the absence of cellular division means that fertilization was "not successful". Fig. 4 shows the average transmission spectra for 3PN with ( 4 oocytes tested) and without division (3 oocytes tested). In this figure we also plot the average spectrum of MII oocytes for comparison purposes. Spectra of 3PN without division are comparable to those of MII oocytes. However, the transmission spectra of 3PN with division are drastically different. These fertilized oocytes become almost transparent, i.e. they become phase objects.

\subsection{Probabilistic behavior of the optical data}

We were aware of the presumed stage of each oocyte. We also knew the value of the minimum transmission. Therefore, for each maturation stage, we construct the histogram of the minimum

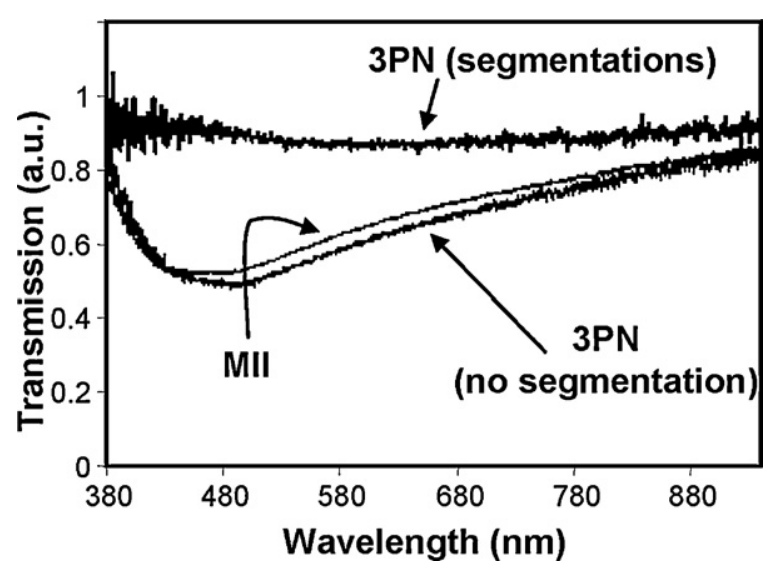

Fig. 4. Experimental results for 3PN oocytes. The 3PN without segmentation and MII oocytes exhibit similar spectra. Spectra of 3PN with segmentation are drastically different.

transmissions. With the histogram, the probability of having one value of the minimum transmission can be calculated. Therefore, for each maturation stage, we can draw the probability density law which is related to the evolutionary process existing in the corresponding maturation stage. The results are shown in Fig. 5(a) for GV, (b) for MI and (c) for MII. Before going any further, let us recall that a Gaussian density corresponds to a pure random process while a uniform density reveals a constant speed evolution process. We chose to consider pure uniform and pure Gaussian densities because they correspond to extreme distributions. Intermediate cases are not yet taken into account, since they would be out of the scope of this study.

The idea is to calculate the probability density attached to each class. In fact, the probability density is not purely Gaussian or uniform. Therefore, we calculated the likeness percentage between the experimental densities and a Gaussian or uniform distribution. The results can be summarized as follows:

- For GV, the probability density is $80 \%$ uniform. This corresponds to an evolutionary process. This means that it is possible to qualify a GV oocyte with a good probability. In fact, the "prefinal $\mathrm{GV}-\mathrm{GV}$ " frontier is well defined and the "GV-MI" frontier is less well defined.

- For MI, the density is $80 \%$ Gaussian, corresponding to a random process. In fact, neither the "GV-MI" nor the "MI-MII" frontier is clearly defined. Therefore the MI class is the most difficult to qualify. This is unsurprising because it corresponds to a transition stage.

- For MII, the density is 50\% uniform and 50\% Gaussian. This class is fairly difficult to qualify. This may be due to the not clearly defined "MI-MII" frontier and the more or less temporal vicinity of the necrosis.

We think that it is necessary to consider the oocytes in their temporal evolution behavior. In order to increase the percentage of successful fertilization, the oocytes must be at the MII stage. However, they may be at the "immature/postmature MII" stage or at the "total/optimal mature MII" stage. Fig. 6 shows the transmission spectra of a single oocyte at 3 points in its maturation evolution. It is typical of the behavior of other oocytes we observed. The time gap between GV and MI is $5 \mathrm{~h}$ compared to $16 \mathrm{~h}$ between MI and MII. The time $T=0$ corresponds to the first observation of the oocyte (at 12 a.m. in this particular case). The vertical arrows are schematic representations of the possible evolution of the oocyte in terms of minimum transmission and wavelength. On the one hand, if the oocyte is supposed to lead to a non-successful fertilization or to 

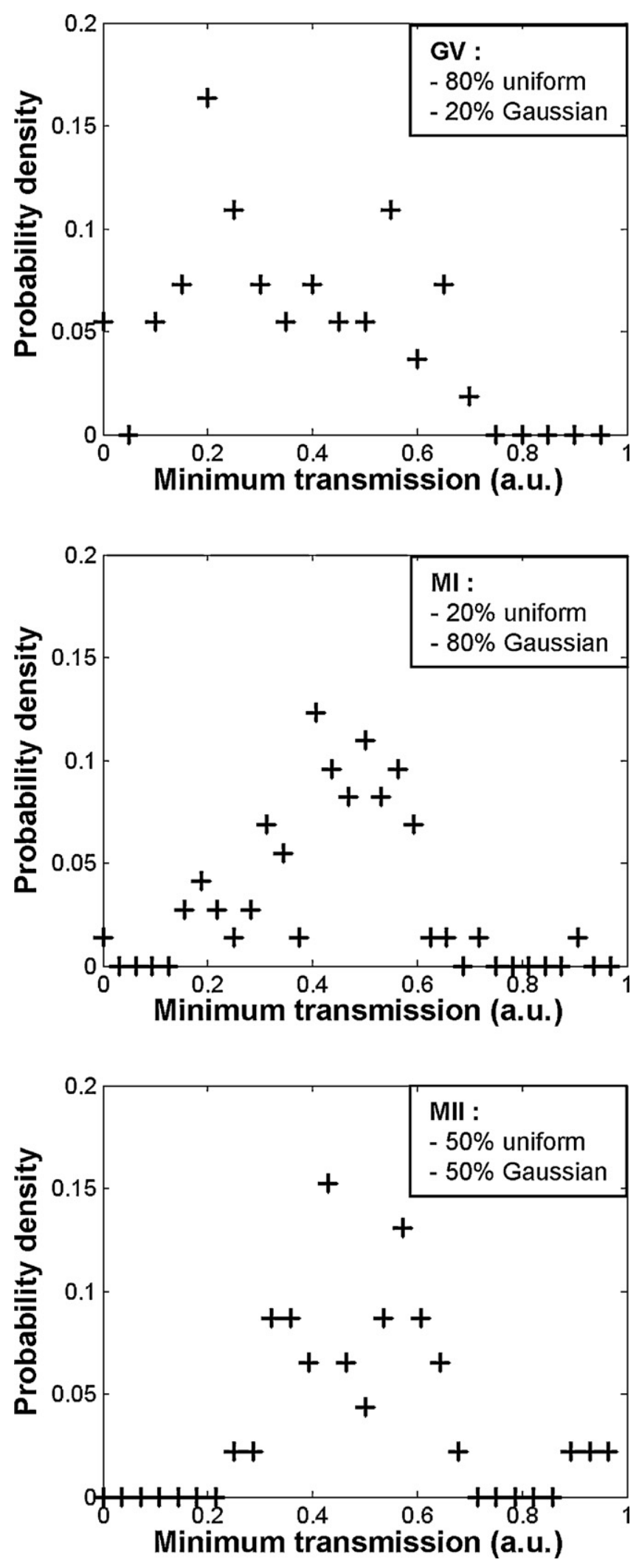

Fig. 5. Probability density laws for GV (top), MI (middle) and MII (bottom) oocytes. The laws are: $80 \%$ uniform $20 \%$ Gaussian for GV, $20 \%$ uniform $80 \%$ Gaussian for MI and $50 \%$ uniform $50 \%$ Gaussian for MII.

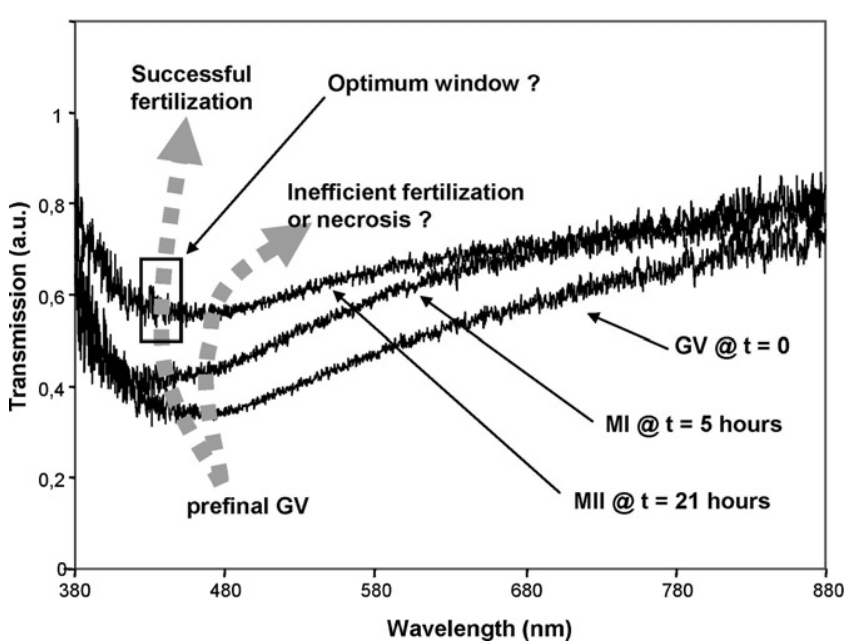

Fig. 6. Temporal evolution of the transmission spectrum for a single oocyte. The oocyte is GV at $t=0, \mathrm{MI}$ at $t=5 \mathrm{~h}$ and MII at $t=21 \mathrm{~h}$. The arrows are schematic representations of any evolution of the oocyte in terms of minimum transmission and wavelength.

necrosis, it follows the arrow on the right. On the other hand, if the oocyte is supposed to lead to a successful fertilization, it follows the arrow on the left during its maturation process.

\section{Discussion}

In this paper we present a simple optical micro-system used to measure the transmission spectra of oocytes. The 3 maturation stages (GV, MI an MII) are easily identified by the transmission spectra analysis. For both parameters, the minimum transmission intensity and the corresponding wavelength, the Student's $t$-test showed that the 3 stages can be identified with a relatively low error probability. However, and as shown by the Box and Whiskers diagrams, although the stages can be distinguished, it does not seem easy to use the transmission spectrum to estimate the maturity of a completely unknown oocyte. It appears difficult to use this method to define the degree of maturity of an oocyte accurately. We find this result unsurprising because the oocytes are subject to a continuous maturation process, from the prefinal GV maturation process to successful fertilization or necrosis in absence of fertilization. Therefore, it is not likely that the transmission spectra differ significantly from one class to another. Of course, 3 maturation stages exist (GV, MI and MII). They correspond to morphological characteristics that can be observed with a microscope.

Visible modifications, GV breakdown and first polar body extrusion cannot alone explain the changes in the spectrum. Through their volume, the changes in cytoplasm and zona pellucida organisation in these final stages contribute to these optical modifications. An analysis of the spectrum of isolated zona pellucida from different stages does not show any difference (data not shown). The changes must therefore be due to cytoplasmic modifications such as migration of cortical granules and mitochondrial redistribution [18].

However, the frontier between two adjacent classes may be difficult to estimate visually. In order to illustrate this, it should be noted that we usually see seven colors in the rainbow. The white light spectrum, when visually observed, apparently exhibits seven different color classes. However, when a spectrogram is recorded, the seven color classes can no longer be distinguished. The qualification of oocytes in 3 classes, which is visually identified by various morphological characteristics, could probably be improved by analysing some of the oocyte properties in a more continuous manner together with the observation of the meiotic event, such as 
spindle formation and its evolution, with the help of a polarization microscope $[19,20]$. This complementary approach can therefore provide information about the modification of the zona pellucida during this maturation process [21]. The analysis of the transmission spectra may be a solution for continuously estimate maturity.

Therefore, in a supposedly normal or abnormal evolution process (Fig. 6) it is likely that an optimum temporal window exists. It is perhaps when the oocyte is in this window that fertilization is the most efficient. In fact, the oocytes should be chosen when they are not only at the MII stage but also at the right time during MII. We think that the use of new qualifying techniques that allow the temporal evolution of the oocytes to be taken into account would be beneficial to IVF techniques. The device we present in this paper is a possibility. However, it should be further improved before it can be used on a routine basis. An alternative technique we presented recently and based on image processing could also be envisaged [17].

In relation to the group of fertilized oocytes for which fertilization abnormalities were observed (3PN oocytes), we showed that the transmission spectra are clearly different between 3PN with segmentation (successful fertilization) and 3PN without segmentation (non-successful fertilization). This impressive change in the transmission spectrum is clearly a sign that important metabolism transformations are occurring. 3PN without segmentation exhibit transmission spectra which are extremely similar to those of MII oocytes. They seem to suffer from cytoplasmic and/or nuclear immaturity. Although they were classified as MII, their particular maturity prevented any further development after fertilization. Perhaps these 3PN were "immature/postmature" MII in cases of absence of segmentation or at the "total/optimum mature" MII stage when segmentation occurred. Fertilization elicits a series of cytological transformations from a morphological and biochemical point of view in the oocyte, such as extrusion of the second polar body, exocytosis of cortical granules, modifications of the zona pellucida as a block to polyspermy, enhanced protein synthesis, intracellular ionic oscillations $\left(\mathrm{Ca}^{2+}\right)$ and pronuclear formation. A disturbance in cytoplasmic events such as a defect in exocytosis of the cortical granules or abnormal hyaloplasmic modifications can be involved in the stagnation of the transmission spectra in 3PN without segmentation [18]. Of course, according to the ethics committee, we did not analyse the transmission spectra of fertilized oocytes exhibiting normal embryo development. However, it is obvious that the analysis of transmission spectra can be considered an interesting fertilization indicator.

\section{Conclusion}

In this paper, we have presented a simple optical micro-system used to measure the transmission spectra of oocytes. It is composed of a micro-machined piece of silicon anodic bonded to a glass substrate. Transmission spectra of oocyte at different maturation stages were recorded and statistical exploitations of the data were proposed. It appears that the system cannot be used to assess the maturity stage (GV, MI and MII) in a blind test.

We think it is not surprising because oocytes continuously evolve from one maturity stage to another one. To draw an interesting conclusion, we believe oocytes should not only be qualified in terms of GV, MI and MII but also regarding their temporal evolution over these maturation stages. The device we propose seems to be able to accurately follow the maturation process of the oocytes in a minimally invasive manner. The next step of our study will be to record the spectra of oocytes more frequently in order to better understand the temporal dynamic of maturation and to obtain additional information about oocyte maturity to assist in future selections of oocyte candidates for ICSI. Embryos must then be chosen in elective single embryo transfer.

\section{Acknowledgments}

This work was supported by a research grant Fond d'aide à la Recherche (FARO) Schering-Plough, France. The authors also would like to thank Frances Sheppard (CIC-IT: Clinical Investigation Center in Technological Innovation of Besançon) for proofreading the article.

\section{References}

[1] D. Loutradis, P. Drakakis, K. Kallianidis, S. Milingos, S. Dendrinos, S. Michalas, Oocyte morphology correlates with embryo quality and pregnancy rate after intracytoplasmic sperm injection, Fertil. Steril. 72 (1999) 240-244.

[2] P.F. Serhal, D.M. Ranieri, A. Kinis, S. Marchant, M. Davies, I.M. Khadum, Oocyte morphology predicts outcome of intracytoplasmic sperm injection, Hum. Reprod. 12 (1997) 1267-1270.

[3] P. Xia, Intracytoplasmic sperm injection: correlation of oocyte grade based on polar body, perivitelline space and cytoplasmic inclusions with fertilization rate and embryo quality, Hum. Reprod. 12 (1997) 1750-1755.

[4] T. Ebner, C. Yaman, M. Moser, M. Sommergruber, O. Feichtinger, G. Tews, Prognostic value of first polar body morphology on fertilization rate and embryo quality in intracytoplasmic sperm injection, Hum. Reprod. 15 (2000) 7-430.

[5] P.M. Ciotti, L. Notarangelo, A.M. Morselli-Labate, V. Felletti, E. Porcu, S. Venturoli, First polar body morphology before ICSI is not related to embryo quality or pregnancy rate, Hum. Reprod. 19 (2004) 2334-2339.

[6] P. De Sutter, D. Dozortsev, C. Qian, Oocyte morphology does not correlate with fertilization rate and embryo quality after intracytoplasmic sperm injection, Hum. Reprod. 11 (1996) 595-597.

[7] B. Balaban, B. Urman, A. Sertac, C. Alatas, S. Aksoy, R. Mercan, Oocyte morphology does no affect fertilization rate, embryo quality and implantation rate after ICSI, Hum. Reprod. 13 (1998) 3431-3433.

[8] R.S. Suh, N. Phadke, D.A. Ohl, S. Takayama, G.D. Smith, Rethinking gamete/embryo isolation and culture with microfluidics, Hum. Reprod. Update 9 (2003) 451-461.

[9] J.F. Danielli, Division of the flattened egg, Nature 170 (1952) 496-1496.

[10] M. Ohtsubo, Y. Hiramoto, Regional differences in mechanical properties of the cell surface in dividing echinoderm eggs, Dev. Growth Differ. 27 (1985) 371-383.

[11] T. Sawai, M. Yoneda, Wave of stiffness propagating along the surface of the newt egg during cleavage, J. Cell Biol. 60 (1974) 1-7.

[12] S. Nakamura, Y. Hiramoto, Mechanical properties of the cell surface in starfish eggs, Dev. Growth Differ. 20 (1978) 317-327.

[13] Y. Murayama, S. Omata, C.E. Constantinou, Micro-mechanical sensing platform for the characterization of the elastic properties of the ovum via uniaxial measurement, J. Biomech. 37 (2004) 67-72.

[14] H. Inui, H. Nakamura, J. Mizuno, Y. Fueta, H. Kamakura, Y. Murayama, S. Omata, K. Akaishi, A. Watanabe, Development of ovum evaluation system for human assisted reproductive technology: verification of the effects and the safety, Hum. Reprod. 1 (Suppl. 1) (2006) i161.

[15] B. Wacogne, C. Pieralli, C. Roux, T. Gharbi, Measuring the mechanical behaviour of human oocytes with a very simple SU-8 micro-tool, Biomed. Microdevices 10 (2008) 411-419.

[16] R. Zeggari, B. Wacogne, C. Pieralli, C. Roux, T. Gharbi, A full micro-fluidic system for single oocyte manipulation including an optical sensor for cell maturity estimation and fertilisation indication, Sens. Actuators B Chem. B 125 (2007) 664-671.

[17] C. Pieralli, B. Wacogne, C. André, C. Roux, C. Joanne, L. Pazart, Biological qualification of oocyte maturity with the use of the Karhunen-Loeve transform: computer aided decision for selecting best oocytes before fertilization, in: Proceedings of the First International Workshop on Medical Image Analysis and Description for Diagnosis Systems, INSTICC Press, ISBN: 978-989-8111-77-7, 2009, pp. 77-84.

[18] A.H. Sathananthan A.H., A.O. Trounson, Mitochondrial morphology during preimplantational human embryogenesis, Hum. Reprod. 15 (Suppl. 2) (2000) $148-159$.

[19] H.T. Zeng, Z. Ren, W.S. Yeung, Y.M. Shu, Y.W. Xu, G.L. Zhuang, X.Y. Liang, Low mitochondrial DNA and ATP contents contribute to the absence of birefringent spindle imaged with PolScope in in vitro matured human oocytes, Hum. Reprod. 6 (2007) 1681-1686.

[20] M. Montag, H. van der Ven, Symposium: innovative techniques in human embryo viability assessment. Oocyte assessment and embryo viability prediction: birefringence imaging, Reprod. Biomed. 4 (2008) 454-460 (online).

[21] M. Montag, T. Schimming, M. Köster, C. Zhou, C. Dorn, B. Rösing, H. van der Ven, $H$. van der Ven, Oocyte zona birefringence intensity is associated with embryonic implantation potential in ICSI cycles, Reprod. Biomed. 2 (2008) 239-244 (online). 